ELECTRONIC RESEARCH ANNOUNCEMENTS OF THE AMERICAN MATHEMATICAL SOCIETY

Volume 8, Pages 11-19 (June 19, 2002)

S $1079-6762(02) 00100-2$

\title{
ON THE CONSTRUCTION OF A $C^{2}$-COUNTEREXAMPLE TO THE HAMILTONIAN SEIFERT CONJECTURE IN $\mathbb{R}^{4}$
}

\author{
VIKTOR L. GINZBURG AND BAŞAK Z. GÜREL \\ (Communicated by Krystyna Kuperberg)
}

\begin{abstract}
We outline the construction of a proper $C^{2}$-smooth function on $\mathbb{R}^{4}$ such that its Hamiltonian flow has no periodic orbits on at least one regular level set. This result can be viewed as a $C^{2}$-smooth counterexample to the Hamiltonian Seifert conjecture in dimension four.
\end{abstract}

\section{INTRODUCTION}

The goal of this notice is to outline the construction of a $C^{2}$-smooth proper function on $\mathbb{R}^{4}$ whose Hamiltonian flow has no periodic orbits on at least one regular level set. A detailed construction of such a function with complete proofs can be found in $[\mathrm{GG}$. This result fits into the circle of questions generally referred to as the Seifert conjecture.

The original Seifert conjecture is the question whether or not there exists a vector field on $S^{3}$ without zeros and periodic orbits. In a broader context, the same question is asked for other manifolds or more restricted classes of vector fields. Traditionally, examples of such vector fields are called counterexamples to the Seifert conjecture. For closed manifolds of dimension greater than three with zero Euler characteristic, $C^{\infty}$-smooth (in fact, real analytic) counterexamples were constructed by Wilson, Wi . A $C^{1}$-smooth counterexample to the Seifert conjecture on $S^{3}$ was found by Schweitzer, $[\mathrm{Sc}]$, and a $C^{1}$-smooth volume-preserving counterexample on $S^{3}$ was constructed by $\mathrm{G}$. Kuperberg, $\mathrm{KuG}$. The ideas from both of these constructions play an important role in this paper. A real analytic counterexample on $S^{3}$ is due to K. Kuperberg, KuGK, KuK1. The reader interested in a detailed survey of the results concerning the original Seifert conjecture should consult KuK2, KuK3.

In a similar vein, the "Hamiltonian Seifert conjecture" is the question whether or not there exists a proper $C^{k}$-function on $\mathbb{R}^{2 n}$ whose Hamiltonian flow has no periodic orbits on at least one regular level set. Hence, the construction outlined here gives a $C^{2}$-counterexample to the Hamiltonian Seifert conjecture in dimension four. (We emphasize that while the function is $C^{2}$, its Hamiltonian vector field is only $C^{1}$-smooth.) In dimensions greater than six, $C^{\infty}$-smooth counterexamples

Received by the editors September 20, 2001.

2000 Mathematics Subject Classification. Primary 37J45; Secondary 53D30.

Key words and phrases. Hamiltonian Seifert conjecture, periodic orbits.

The work is partially supported by the NSF and by the faculty research funds of the University of California, Santa Cruz. 
to the Hamiltonian Seifert conjecture were constructed by M. Herman, He1, He2, and simultaneously by one of the authors, Gi1. In dimension six, a $C^{2+\epsilon}$-smooth counterexample was found by M. Herman, $\mathrm{He} 1 \mathrm{He} 2$. This smoothness constraint was later relaxed to $C^{\infty}$ in Gi2. A very simple and elegant construction of new $C^{\infty}$ smooth counterexamples in dimensions greater than four was recently discovered by Kerman, $\mathrm{Ke}$. We refer the reader to Gi3 Gi4 for a detailed discussion of the Hamiltonian Seifert conjecture.

An essential difference of the Hamiltonian case from the general one is manifested by the almost existence theorem, [HZ1, HZ2, St], which asserts that almost all regular levels of a proper Hamiltonian have periodic orbits. To be more specific, for a $C^{2}$-smooth (and probably even $C^{1}$-smooth) proper Hamiltonian on $\mathbb{R}^{2 n}$ regular values without periodic orbits form a zero measure set. Thus such regular levels are exceptional in the sense of measure theory.

Acknowledgments. The authors are deeply grateful to Helmut Hofer, Anatole Katok, Ely Kerman, Krystyna Kuperberg, Mark Levi, Debra Lewis, Rafael de la Llave, Eric Matsui, and Maria Schonbek for useful discussions and suggestions.

\section{MAin RESUlts}

Recall that the characteristic foliation on a hypersurface $M$ in a symplectic manifold $(W, \eta)$ is the one-dimensional foliation whose leaves (called characteristics) are tangent to the field of directions $\operatorname{ker}\left(\left.\eta\right|_{M}\right)$.

Let $\mathbb{R}^{2 n}$ be equipped with its standard symplectic structure.

Theorem 2.1. There exists a $C^{2}$-smooth embedding $S^{3} \hookrightarrow \mathbb{R}^{4}$ such that its characteristic foliation has no closed characteristics. This embedding can be chosen $C^{0}$-close and $C^{2}$-isotopic to an ellipsoid.

As an immediate consequence we obtain

Theorem 2.2. There exists a proper $C^{2}$-function $F: \mathbb{R}^{4} \rightarrow \mathbb{R}$ such that the level $\{F=1\}$ is regular and has no periodic orbits. In addition, $F$ can be chosen so that this level is $C^{0}$-close and $C^{2}$-isotopic to an ellipsoid.

Note that since all values of $F$ near $F=1$ are regular, by the almost existence theorem, almost all levels of $F$ near this level carry periodic orbits.

Remark 2.3. It is likely that our construction of the embedding $S^{3} \hookrightarrow \mathbb{R}^{4}$ gives in fact a $C^{2+\alpha}$-embedding without closed characteristics. However, checking this requires considerably more work than needed to verify $C^{2}$-smoothness.

Remark 2.4. Similarly to its higher-dimensional counterparts, Gi1, Gi2, Theorem 2.1 extends to other symplectic manifolds as follows. Let $(W, \eta)$ be a fourdimensional symplectic manifold, and let $i: M \hookrightarrow W$ be a $C^{\infty}$-smooth embedding such that $i^{*} \eta$ has only a finite number of closed characteristics. Then there exists a $C^{2}$-smooth embedding $i^{\prime}: M \hookrightarrow W$, which is $C^{0}$-close and isotopic to $i$, such that $i^{\prime *} \eta$ has no closed characteristics.

In what follows we outline the proof of Theorem 2.1. The idea of the proof is to adjust Schweitzer's construction, $\underline{\mathrm{Sc}}$, of a $C^{1}$-flow on $S^{3}$ without periodic orbits to make it embeddable into $\mathbb{R}^{4}$ as a Hamiltonian flow. This is done by constructing a version of Schweitzer's plug with suitable properties similar to those of G. Kuperberg's plug, $\underline{\mathrm{KuG}}$. The essential feature of the construction is that 
Schweitzer's plug has to be built using a "very smooth" Denjoy flow (see Remark 4.2).

Remark 2.5. As of this moment we do not know if G. Kuperberg's flow can be embedded into $\mathbb{R}^{4}$. The two constructions differ in an essential way. The Denjoy flow in G. Kuperberg's example is required to have properties very different from the ones we need. As a consequence, our method to embed the plug into $\mathbb{R}^{4}$ does not apply to G. Kuperberg's plug. (For example, one technical but essential discrepancy between the constructions is as follows. In G. Kuperberg's construction, it is important to take a rotation number which cannot be too rapidly approximated by rationals, while the Denjoy map is not required to be smoother than just $C^{1}$. On the other hand, in our construction the value of rotation number is irrelevant, but the smoothness of the Denjoy map plays a crucial role.)

\section{Construction of Symplectic EMBedding}

Let us first fix some notation. Throughout this paper $\sigma$ denotes the standard symplectic form on $\mathbb{R}^{2 m}$ or the pullback of this form to $\mathbb{R}^{2 m+1}$ by the projection $\mathbb{R}^{2 m+1} \rightarrow \mathbb{R}^{2 m}$ along the first coordinate; $I^{2 m}$ stands for a cube in $\mathbb{R}^{2 m}$ whose edges are parallel to the coordinate axes. The product $[a, b] \times I^{2 m}$ is always assumed to be embedded into $\mathbb{R}^{2 m+1}$ (henceforth, the standard embedding) so that the interval $[a, b]$ is parallel to the first coordinate. We refer to the direction along the first coordinate $t$ (time) in $\mathbb{R}^{2 m+1}$ (or $[a, b]$ in $[a, b] \times I^{2 m}$ ) as the vertical direction. All maps whose smoothness is not specified are $C^{\infty}$-smooth.

Theorem 2.1 follows, as do similar theorems in dimensions greater than four, from the existence of a symplectic plug. The definitions of a plug vary considerably (see [Gi1, $\mathrm{Ke}, \mathrm{KuG}]$ ), and here we use the one more suitable for our purposes.

A $C^{k}$-smooth symplectic plug in dimension $2 n$ is a $C^{k}$-embedding $J$ of $P=$ $[a, b] \times I^{2 n-2}$ into $P \times \mathbb{R} \subset \mathbb{R}^{2 n}$ such that

(P1) The boundary condition: The embedding $J$ is the identity embedding of $P$ into $\mathbb{R}^{2 n-1}$ near the boundary $\partial P$. Thus the characteristics of $J^{*} \sigma$ are parallel to the vertical direction near $\partial P$.

(P2) Aperiodicity: The characteristic foliation of $J^{*} \sigma$ is aperiodic, i.e., it has no closed characteristics.

(P3) Existence of trapped trajectories: There is a characteristic of $J^{*} \sigma$ beginning on $\{a\} \times I^{2 n-2}$ that never exits the plug. Such a characteristic is said to be trapped in $P$.

(P4) The embedding $J$ is $C^{0}$-close to the standard embedding and $C^{k}$-isotopic to it.

(P5) Matched ends condition: For a characteristic that meets both the bottom and the top of the plug, its top end lies exactly above the bottom point.

Theorem 3.1. In dimension four, there exists a $C^{2}$-smooth symplectic plug.

Theorem 2.1 readily follows from Theorem 3.1 by applying a symplectic plug to perturb an irrational ellipsoid in $\mathbb{R}^{4}$.

Proof of Theorem [3.1. By the standard symmetry argument it suffices to construct a semiplug, i.e., "plug" satisfying only conditions $(\mathrm{P} 1)-(\mathrm{P} 4)$. We will construct a semiplug by perturbing the natural embedding of $[a, b] \times I^{2}$ into $\mathbb{R}^{4}$. 
Let $\Sigma$ be the torus $T^{2}$ with coordinates $(x, y)$, punctured at $\left(x_{0}, y_{0}\right)$, i.e., with a neighborhood of $\left(x_{0}, y_{0}\right)$ deleted. It is easy to see that there exists an embedding

$$
M=[-1,1] \times \Sigma \hookrightarrow[a, b] \times I^{2} \subset \mathbb{R}^{3} \subset \mathbb{R}^{4},
$$

referred to in what follows as the standard embedding, such that the pullback of $\sigma$ is $d x \wedge d y$. From now on, we will identify $M$ with the image of this embedding.

The embedding $J$ is obtained from the standard embedding by altering it within $M$. This is done in a few steps. First, we consider an embedding of $M$ into some four-dimensional symplectic manifold $\left(W, \sigma_{W}\right)$ such that the pullback of $\sigma_{W}$ is still $d x \wedge d y$. Then we $C^{0}$-perturb this embedding so that the characteristic vector field of the new pullback will have the properties similar to those of Schweitzer's plug. By the symplectic neighborhood theorem, a neighborhood of $M$ in $W$ is symplectomorphic to that of $M$ in $\mathbb{R}^{4}$. This will allow us to turn the embedding $M \hookrightarrow W$ into the required embedding $J: M \hookrightarrow \mathbb{R}^{4}$.

As in Schweitzer's construction, the characteristic vector field of $J$ is a modification of a Denjoy vector field on $T^{2}$. This modification is obtained by extending the Denjoy vector field to $[-1,1] \times T^{2}$ and then restricting it to $\varphi(M)$ for a suitable embedding $\varphi: M \rightarrow[-1,1] \times T^{2}$. Likewise, $J$ is essentially equal to $j \varphi$ for an embedding $j$ of $[-1,1] \times T^{2}$ into $W$. Hence we start with defining $j$ on $[-1,1] \times T^{2}$.

Let $W=(-2,2) \times S^{1} \times T^{2}$ with coordinates $(t, x, u, y)$ and symplectic form

$$
\sigma_{W}=d t \wedge d x+d u \wedge d y
$$

and let

$$
j_{0}:[-1,1] \times T^{2} \hookrightarrow W ; \quad j_{0}(t, x, y)=(t, x, x, y) .
$$

Consider a mapping $K:[-1,1] \times T^{2} \rightarrow S^{1}$ to be specified later on and define

$$
j:[-1,1] \times T^{2} \rightarrow(-2,2) \times S^{1} \times T^{2} \quad \text { by } j(t, x, y)=(t, x, K, y) .
$$

(To illustrate this definition note that when $K(t, x, y)=x$, the embedding $j$ turns into $j_{0}$.) The characteristic vector field of the pullback form $j^{*} \sigma_{W}$ is

$$
v=\left(\partial_{x} K\right) \partial_{t}-\left(\partial_{t} K\right) \partial_{x}+\partial_{y} .
$$

Remark 3.2. To justify the definition of $j$, let us view the annulus $[-1,1] \times S^{1}$ as a symplectic manifold with symplectic form $d t \wedge d x$, and the product $[-1,1] \times T^{2}$ as the extended phase space with the $y$-coordinate being the time variable. Then we can regard $K$ as a (multivalued) time-dependent Hamiltonian on $[-1,1] \times S^{1}$ and view $W$ as the further extended time-energy phase space with the cyclic energy coordinate $u$. Then $j$ is the graph of the time-dependent Hamiltonian $K$ in the extended time-energy phase space $W$. Now it is clear that $v$ is just the Hamiltonian vector field of $K$.

In what follows we choose $\varphi: M \rightarrow[-1,1] \times T^{2}$ so as to make a neighborhood $U$ of $j_{0} \varphi(M)$ in $W$ symplectomorphic to a neighborhood of $M$ in $\mathbb{R}^{4}$. Then, if $j$ takes values in $U$ and matches $j_{0}$ near the boundary $\partial M$, we can turn $j \varphi$ into the desired embedding $J$. However, to ensure that (P1)-(P4) are satisfied we need to impose some requirements on $K$. Let us now describe these requirements.

Let $\partial_{y}+h \partial_{x}$ be a Denjoy vector field on $T^{2}$ to be specified in Section 4 , and let $\mathfrak{D}$ be the Denjoy continuum for this field. (See, e.g., $[\mathrm{KH}, \mathrm{Sc}]$ for an introduction to Denjoy maps and vector fields.) Here we only point out that $\left(x_{0}, y_{0}\right)$ is chosen in the complement of $\mathfrak{D}$. Fix a small neighborhood $V$ of $\left(x_{0}, y_{0}\right)$ disjoint from $\mathfrak{D}$. 
(This neighborhood will contain the puncture, i.e., $\partial \Sigma \subset V$.) Consider the tubular neighborhood of the line $\left(t, x_{0}, y_{0}+t\right)$ in $[-1,1] \times T^{2}$ of the form

$$
\{(t, x, y+t) \mid(x, y) \in V, t \in[-1,1]\} .
$$

Fix also a small neighborhood of the boundary $\partial\left([-1,1] \times T^{2}\right)$ and denote by $N$ the union of these neighborhoods.

Proposition 3.3. There exists a $C^{2}$-smooth mapping $K:[-1,1] \times T^{2} \rightarrow S^{1}$ such that

(K1) $v$ is equal to the Denjoy vector field (i.e., $\partial_{x} K=0$ and $\partial_{t} K=-h$ ) at every point of $\{0\} \times \mathfrak{D}$;

(K2) the $t$-component of $v$ is positive (i.e., $\partial_{x} K>0$ ) on the complement of $\{0\} \times \mathfrak{D}$;

(K3) $K$ is $C^{0}$-clos 11 to the map $K_{0}:(t, x, y) \mapsto x$;

(K4) $K=K_{0}$ on $N$.

Let us defer the proof of this proposition to Section 4 and finish the proof of Theorem 3.1. From now on we assume that $K$ is as in Proposition 3.3

By (K1) and (K2), $v$ has a trapped trajectory. Otherwise, there would exist $\tau>0$ such that every point at the bottom of the plug reaches the top of the plug in a time less than $\tau$. This, however, is impossible since points passing close to the invariant set $\{0\} \times \mathfrak{D}$ spend arbitrarily long time in a neighborhood of this set.

Furthermore, $v$ is aperiodic. Indeed, by (K1), $\{0\} \times \mathfrak{D}$ is invariant under the flow of $v$, and on this set the flow is a Denjoy flow. By (K2), the vertical component of $v$ is nonzero unless the point is in $\{0\} \times \mathfrak{D}$. This implies that periodic orbits can only occur within $\{0\} \times \mathfrak{D}$. Since the Denjoy flow is aperiodic, so is the entire flow of $v$.

Let

$$
\varphi: M=[-1,1] \times \Sigma \rightarrow[-1,1] \times T^{2} ; \quad \varphi(t, x, y)=(t, x, y+t) .
$$

Then $\left(j_{0} \varphi\right)^{*} \sigma_{W}=d x \wedge d y$. The argument similar to the proof of the symplectic neighborhood theorem, [McDS, Lemma 3.14], shows that a "neighborhood" of $M$ in $\mathbb{R}^{4}$ is symplectomorphic to a "neighborhood" $U$ of $j_{0} \varphi(M)$ in $W$ (see Gi1] Section 4] and [GG] for details). More precisely, for a small $\delta>0$, there exists a symplectomorphism

$$
\psi: M \times(-\delta, \delta) \rightarrow U \subset W
$$

extending $j_{0} \varphi$, i.e., such that $\left.\psi\right|_{M}=j_{0} \varphi$.

By (K3), $j$ is $C^{0}$-close to $j_{0}$ and $j=j_{0}$ on $N$ by (K4). Hence, $j$ can be assumed to take values in $U$. Set

$$
J=\psi^{-1} j \varphi
$$

on $M$ and extend $J$ as the standard embedding to $[a, b] \times I^{2} \backslash M$. Then $\left.\left(J^{*} \sigma\right)\right|_{M}=$ $(j \varphi)^{*} \sigma_{W}$.

The characteristic vector field of $J^{*} \sigma$ is $\partial_{t}$ in the complement of $M$ and $\left(\varphi^{-1}\right)_{*} v$ on $M$. Since $\left(\varphi^{-1}\right)_{*} v=\partial_{t}$ near $\partial M$, these vector fields match smoothly at $\partial M$. Clearly, (P1) is satisfied. Since $v$ has a trapped trajectory and is aperiodic, the same is true for $\left(\varphi^{-1}\right)_{*} v$, i.e., the conditions (P2) and (P3) are met. The condition (P4) is easy to verify. Hence, $J$ is a semiplug.

\footnotetext{
${ }^{1}$ More specifically, for any $\epsilon>0$ there exists a $K$ satisfying (K1)-(K2) and (K4) such that $\left\|K-K_{0}\right\|<\epsilon$. The required value of $\epsilon$ is determined by the size of the neighborhood $U$ in the symplectic neighborhood theorem; see below.
} 
Remark 3.4. In the proof of Proposition 3.3 we will not require the Denjoy continuum $\mathfrak{D}$ to have zero measure. As a consequence, the union of characteristics entirely contained in the semiplug can have Hausdorff dimension two because this set is the image of $\mathfrak{D}$ under a $C^{2}$-smooth embedding.

\section{Construction of the function $K$}

4.1. Denjoy vector field. The first step in the construction of $K$ is to choose an appropriate Denjoy vector field on $T^{2}$.

Lemma 4.1. There exists a Denjoy vector field $\partial_{y}+h \partial_{x}$ which is $C^{1+\alpha}$ for all $\alpha \in(0,1)$ and such that

(D1) $\partial_{x} h$ vanishes on the Denjoy continuum $\mathfrak{D}$;

(D2) $\int_{0}^{x}\left(\partial_{x} h(\xi, y)\right)^{2} d \xi$ is $C^{2}$ in $(x, y)$.

Sketch of the proof of Lemma 4.1. We start with the Denjoy map $\Phi: S^{1} \rightarrow S^{1}$ defined using intervals $I_{n}$ of length

$$
l_{n}:=k_{\beta}(|n|+2)^{-1}(\log (|n|+2))^{-1 / \beta}
$$

for some $\beta \in(0,1)$. Here $k_{\beta}$ is a constant depending on $\beta$ chosen so that $\sum_{n \in \mathbb{Z}} l_{n}<$ 1. (See $[\mathrm{KH}]$ for details.) The importance of our choice of $l_{n}$ is that the series $\sum_{n \in \mathbb{Z}} l_{n}$ converges very slowly, which results in a small Denjoy continuum $\mathfrak{D}_{0}=$ $S^{1} \backslash \bigcup_{n \in \mathbb{Z}} \operatorname{Int}\left(I_{n}\right)$. This slow convergence is the main factor which ensures that $\Phi$ is sufficiently smooth. More specifically, it is well known and easy to see that $\Phi$ is $C^{1+\alpha}$ for any $\alpha \in(0,1)$ regardless of what $\beta$ we have taken. Furthermore, we claim that

$$
\left(\Phi^{\prime}-1\right)^{2} \quad \text { is } \quad C^{1} .
$$

Indeed, $\Phi^{\prime}-1$ vanishes on $\mathfrak{D}_{0}$. Then the fact that $\Phi^{\prime}-1$ is $C^{\alpha}$ with $\alpha>1 / 2$ readily implies that $\left(\Phi^{\prime}-1\right)^{2}$ is everywhere differentiable and that its derivative vanishes along $\mathfrak{D}_{0}$. The proof that this derivative is continuous relies explicitly on (1). Using (11), one can show that

$$
\left\|\left.\frac{d}{d x}\left(\Phi^{\prime}-1\right)^{2}\right|_{I_{n}}\right\|_{\infty} \rightarrow 0 \quad \text { as } \quad n \rightarrow \infty
$$

which ensures the continuity of the derivative.

Now let $\partial_{x}+h \partial_{y}$ be a suitably defined Denjoy vector field on $T^{2}$ for $\Phi$. The $C^{1+\alpha}$-smoothness of $h$ and (D1) do not present a problem. However, the verification of (D2) is more involved. On the conceptual level, the proof is similar to that of (2) outlined above. To illustrate this point, observe that $\Phi^{\prime}-1$ can be taken as an analogue of $\partial_{x} h$, and one may expect these two functions to have the same smoothness properties. (In fact, $\left(\partial_{x} h\right)^{2}$ is $C^{1}$ in the $x$-variable.) The actual proof of (D2) requires a direct verification of the existence and continuity of derivatives using their asymptotic behavior on the intervals $I_{n}$; see GG] for details.

Remark 4.2. The Denjoy map defined by (1) is essentially as smooth as a Denjoy map can be, up to using functions growing more slowly than logarithms, e.g., iterations of logarithms. The next significant improvement in smoothness would be to have $\log \Phi^{\prime}$ of bounded variation or satisfying the Zygmund condition, which is impossible; see [HS, $\mathrm{KH}]$. 
4.2. Construction of K. First we extend $h$ to a $C^{1}$-function on $H:[-1,1] \times T^{2} \rightarrow$ $\mathbb{R}$ so that

$$
\partial_{x} H(t, x, y)=\partial_{x} h(x, y)+o(t) \quad \text { uniformly in }(x, y)
$$

and the function

$$
\int_{0}^{t} H(\tau, x, y) d \tau \quad \text { is } C^{2} \text { in }(t, x, y) .
$$

The value $H(t, x, y)$ is obtained by averaging $h$ over the square with side $t^{s}$, for some $s>0$, centered at $(x, y)$. (This averaging argument is somewhat similar to the one in $\left[\mathrm{KuG}\right.$.) Then using only the fact that $h$ is $C^{1+\alpha}$ with $\alpha>1 / 2$, it is not hard to show that $H$ satisfies (3) and (4).

Now we are in a position to define the function $K$. We focus on a small neighborhood $U$ of $\mathfrak{D}$ in $T^{2}$ and small values of $t$. In other words, $K$ will be defined only on $[-\epsilon, \epsilon] \times U$, where $\epsilon>0$ is sufficiently small. Furthermore, we consider only the requirements (K1) and (K2). For this is where the essence of the problem lies, whereas the conditions (K3) and (K2) are purely technical. With these restrictions on the domain of $K$ and the hypotheses this function should satisfy, we can take $K$ to be real-valued rather than circle-valued. (We refer the reader to [GG] for a complete construction of $K$ and a detailed proof of (K1)-(K4).)

We will construct the function $K$ of the form

$$
K(t, x, y)=-\int_{0}^{t} H(\tau, x, y) d \tau+A(x, y)+f(x, y) t^{2} .
$$

Here the "constant" of integration $A$ is a $C^{2}$ function $U \rightarrow \mathbb{R}$ such that

$$
\partial_{x} A \geq 2\left(\partial_{x} h\right)^{2} \quad \text { and } \quad \partial_{x} A(x, y)=0 \quad \text { iff } \quad(x, y) \in \mathfrak{D} .
$$

The existence of such a function depends heavily on (D1) and (D2). Indeed, $\partial_{x} A$ is $C^{1}$, and $\partial_{x} A$ and $\left(\partial_{x} h\right)^{2}$ both vanish on $\mathfrak{D}$. Thus, (6) forces $\left(\partial_{x} h\right)^{2}$ to be at least differentiable in $x$ at the points of $\mathfrak{D}$. Conversely, using (D1) and (D2), we can set

$$
A(x, y)=2 \int_{0}^{x}\left(\partial_{x} h(\xi, y)\right)^{2} d \xi+a(x, y)
$$

where $a$ is a $C^{\infty}$-function on $U$ such that $\partial_{x} a \geq 0$ and $\partial_{x} a=0$ exactly on $\mathfrak{D}$. Then $A$ will satisfy (6).

The correction function $f: U \rightarrow \mathbb{R}$ is $C^{\infty}$-smooth and chosen so that

$$
\partial_{x} f=2
$$

(One can show that such a function does exist.)

Let us now verify the properties of $K$. First note that $K$ is indeed $C^{2}$-smooth by (4). Condition (K1) is also obvious: since $\left.H\right|_{t=0}=h$, we have $\left.\partial_{t} K\right|_{t=0}=-\left.H\right|_{t=0}=$ $-h$ and $\left.\partial_{x} K\right|_{\{0\} \times \mathfrak{D}}=\left.\partial_{x} A\right|_{\mathfrak{D}}=0$ by (6).

Let us now turn to (K2). We will first show that

$$
\partial_{x} K=-\int_{0}^{t} \partial_{x} H d \tau+\partial_{x} A+2 t^{2} \geq 0
$$

and then prove that the equality occurs only on $\{0\} \times \mathfrak{D}$.

By (3), we have

$$
\partial_{x} K=\partial_{x} A-t \partial_{x} h+t^{2}+\left(t^{2}+o\left(t^{2}\right)\right) .
$$


Clearly,

$$
t^{2}+o\left(t^{2}\right) \geq 0
$$

if $\epsilon>0$ is small. Hence, to verify (17) it suffices to show that

$$
\partial_{x} A-t \partial_{x} h+t^{2} \geq 0 \text {. }
$$

By (6)), this inequality is a consequence of

$$
2\left(\partial_{x} h\right)^{2}-t \partial_{x} h+t^{2}=\left(\frac{\partial_{x} h}{2}-t\right)^{2}+\frac{7}{4}\left(\partial_{x} h\right)^{2} \geq 0 .
$$

To finish the proof of (K2) we need to show that the equality in (7) implies that $t=0$ and $(x, y) \in \mathfrak{D}$. Thus, assume that $\partial_{x} K(t, x, y)=0$. Then (8) and (9) must become equalities. The equality (8) is possible only when $t=0$. Setting $t=0$ in the equality (9), we conclude that $\partial_{x} A(x, y)=0$ and hence $(x, y) \in \mathfrak{D}$ by (6). This concludes the proof of (K2).

Remark 4.3. The actual construction of $K$, and, in particular, of $A$ and $f$, given in [GG] differs considerably from the one above. In fact, when $K$ is defined on $[-\epsilon, \epsilon] \times T^{2}$, the constant of integration $A$ is a function $T^{2} \rightarrow S^{1}$. Moreover, some extra care is needed in the definition of $f$. However, these difficulties are rather technical, and our description captures well the main idea of the definition of $K$.

\section{REFERENCES}

[Gi1] V. L. Ginzburg, An embedding $S^{2 n-1} \rightarrow \mathbb{R}^{2 n}, 2 n-1 \geq 7$, whose Hamiltonian flow has no periodic trajectories, IMRN 1995, no. 2, 83-98. MR 96a:58087

[Gi2] V. L. Ginzburg, A smooth counterexample to the Hamiltonian Seifert conjecture in $\mathbb{R}^{6}$, IMRN 1997, no. 13, 641-650. MR 98i:58087

[Gi3] V. L. Ginzburg, Hamiltonian dynamical systems without periodic orbits, in Northern California Symplectic Geometry Seminar, pp. 35-48, Amer. Math. Soc. Transl. Ser. 2, vol. 196, Amer. Math. Soc., Providence, RI, 1999. MR 2001c:37055

[Gi4] V. L. Ginzburg, The Hamiltonian Seifert conjecture: examples and open problems, math.DG/0004020; to appear in Proceedings of the Third ECM, Barcelona, 2000, Birkhäuser.

[GG] V. L. Ginzburg and B. Z. Gürel, A $C^{2}$-smooth counterexample to the Hamiltonian Seifert conjecture in $\mathbb{R}^{4}$, in preparation.

[He1] M.-R. Herman, Fax to Eliashberg, 1994.

[He2] M.-R. Herman, Examples of compact hypersurfaces in $\mathbb{R}^{2 p}, 2 p \geq 6$, with no periodic orbits, in Hamiltonian systems with three or more degrees of freedom, C. Simo (Editor), NATO Adv. Sci. Inst. Ser. C, Math. Phys. Sci., vol. 533, Kluwer Acad. Publ., Dordrecht, 1999. MR 2000f:37004

[HZ1] H. Hofer and E. Zehnder, Periodic solution on hypersurfaces and a result by C. Viterbo, Invent. Math. 90 (1987), 1-9. MR 89a:58040

[HZ2] H. Hofer and E. Zehnder, Symplectic invariants and Hamiltonian dynamics, Birkhäuser, Advanced Texts; Basel-Boston-Berlin, 1994. MR 96g:58001

[HS] J. Hu and D. Sullivan, Topological conjugacy of circle diffeomorphisms, Ergodic Theory Dynam. Systems 17 (1997), 173-186. MR 97m:58122

$[\mathrm{KH}]$ A. Katok and B. Hasselblatt, Introduction to the modern theory of dynamical systems, Encyclopedia of Mathematics and its Applications, vol. 54. Cambridge University Press, Cambridge, 1995. MR 96c:58055

[Ke] E. Kerman, New smooth counterexamples to the Hamiltonian Seifert conjecture, Preprint 2001, math.DG/0101185; to appear in the Journal of Symplectic Geometry.

[KuG] G. Kuperberg, A volume-preserving counterexample to the Seifert conjecture, Comment. Math. Helv. 71 (1996), 70-97. MR 96m:58199

[KuGK] G. Kuperberg and K. Kuperberg, Generalized counterexamples to the Seifert conjecture, Ann. Math. 144 (1996), 239-268. MR 97k:57031b 
[KuK1] K. Kuperberg, A smooth counterexample to the Seifert conjecture in dimension three, Ann. Math. 140 (1994), 723-732. MR 95g:57040

[KuK2] K. Kuperberg, Counterexamples to the Seifert conjecture, Proceedings of the International Congress of Mathematicians, Vol. II (Berlin, 1998). Doc. Math. (1998), Extra Vol. II, 831-840. MR 99j:58163

[KuK3] K. Kuperberg, Aperiodic dynamical systems, Notices Amer. Math. Soc. 46 (1999), 10351040. MR 2000f:37024

[McDS] D. McDuff and D. Salamon, Introduction to symplectic topology, Oxford Mathematical Monographs, Oxford University Press, New York, 1995. MR 97b:58062

[Sc] P.A. Schweitzer, Counterexamples to the Seifert conjecture and opening closed leaves of foliations, Ann. Math. 100 (1970), 229-234. MR 50:8557]

[St] M. Struwe, Existence of periodic solutions of Hamiltonian systems on almost every energy surface, Bol. Soc. Bras. Mat. 20 (1990), 49-58. MR 92k:58225

[Wi] F. Wilson, On the minimal sets of nonsingular vector fields, Ann. Math. 84 (1966), 529-536. MR 34:2028

Department of Mathematics, UC Santa Cruz, Santa Cruz, CA 95064, USA

E-mail address: ginzburg@math.ucsc.edu

Department of Mathematics, UC Santa Cruz, Santa Cruz, CA 95064, USA

E-mail address: basak@math.ucsc.edu 